\title{
Continuous Measurements of Tissue Impedance during Secretion in Dog Submandibular Gland
}

\author{
Takashi NaKahari, Manabu MiYamoto, Hideyo Yoshida, \\ Haruo TANAKA, and Yusuke IMAI \\ Department of Physiology, Osaka Medical College, \\ Takatsuki, Osaka, 569 Japan
}

\begin{abstract}
The electrical impedance of the dog submandibular gland, as an indicator of changes in extracellular fluid (ECF) volume, was measured at $5 \mathrm{kHz}, 500 \mathrm{kHz}$, and $5 \mathrm{MHz}$ at intervals of $10 \mathrm{~s}$ during secretory stimulation, because the conductivity calculated from impedance at low frequencies reflected the ECF volume. The decrease in conductivity occurred in the first minute of stimulation. Its decrease was more marked during stimulation after circulatory arrest. Salivary secretion under intact circulation consists of two phases: an initial secretion occurring in the first minute of stimulation with a high secretory rate and a steady secretion continuing during stimulation at a constant rate. A decrease in conductivity occurred in the initial secretion. Within a few minutes following the cessation of stimulation, the conductivity increased to a level higher than the resting one with an intact circulation, while the blood flow remained several times higher than in the resting state. The conductivity of the gland slowly recovered to the prestimulation level over a 30 -min period. The histological examination revealed that the main compartment of the ECF change was the interlobular space. Impedance and histological studies showed that the ECF volume of salivary glands changes dynamically during secretion. On the other hand, the cellular volume also increased in the initial secretion of an isolated gland. Its increase suggested that the fluid was transported to the lumen via a cellular pathway as well as a paracellular pathway in the initial secretion.
\end{abstract}

Key words: extracellular fluid, salivary gland, impedance, salivary secretion, conductivity.

Tissue impedance measured over a wide frequency range is useful for continuous and noninvasive monitoring of extracellular fluid (ECF) volume (NAKAHARI et al., 1986a, b), although there are some technical difficulties, such as electrode polarization and depressed circle on the Cole-Cole plot (SCHWAN, 1963; ColE, 1968;

Received for publication September 24, 1987 
AsAmi and IRIMAJIRI, 1984; Asami et al., 1984). In cell suspensions, the cellular volume fraction has already been computed from the conductivity of the suspension and the suspending fluid (IRIMAJRI et al., 1975). This follows from the study of HANAI (1968) on emulsions. In this study, the decreasing or increasing process of glandular ECF was continuously monitored during secretory stimulation under the assumption that the equation of Hanai on cell suspension is applicable to the submandibular gland of dogs.

\section{METHODS}

a) Submandibular gland preparation. Dogs were anesthetized by intravenous administration of thiopental sodium $(40-50 \mathrm{mg} / \mathrm{kg})$. The surgical procedure has been described previously (NAKAHARI et al., 1985a). The experiment was performed under in situ condition. The chorda tympani was isolated and its peripheral end was stimulated $(5 \mathrm{~V} ; 20 \mathrm{~Hz}$; pulse duration, $3 \mathrm{~ms})$. The blood flow through the gland and secreted saliva were measured continuously by electronic balances (MURAKAMI et al., 1980). To achieve circulatory arrest, the artery was clamped. The dog was placed on a rubber sheet for insulation from the ground. After the experiment, the gland was isolated for weighing.

In separate experiments, isolated submandibular glands were also used. Chorda tympani stimulation was performed, and secreted saliva was monitored continuously. The gland was weighed before and after experiments.

b) Impedance measurements. The electrodes, a pair of platinum disks $(5 \mathrm{~mm}$ in diameter) with platinum black-coated surfaces, were directly attached to the submandibular gland.

In cell suspension the correlation between the cellular volume fraction and the measured impedance is represented by the following equation on the assumption that the conductivity of the cell membrane is negligible compared with those of the intra- and extracellular fluids:

$$
\kappa / \kappa_{\mathrm{m}}=(1-\phi)^{3 / 2}
$$

where $\kappa$ and $\kappa_{\mathrm{m}}$ are conductivities of a cell suspension and a cell-free medium, and $\phi$ is cellular volume fraction. This equation holds for low frequencies. The admittance of the submandibular gland was measured by a YHP 4192A LF Impedance Analyzer in 3 frequencies $(5 \mathrm{kHz}, 500 \mathrm{kHz}, 5 \mathrm{MHz})$ at intervals of $10 \mathrm{~s}$. The residual inductance effect of the circuit was corrected (AsAmI et al., 1984), and the data were expressed as the conductivity and relative permittivity, as mentioned previously (NAKAHARI et al., 1986b). From these values, resistance and reactance were calculated, and plotted in a complex plane (Cole-Cole plot).

The data were fitted to an arc expressed in the following equation (COLE and CoLe, 1941):

$$
\left.Z^{*}=r_{0}+r_{1} /(1+j \omega \tau)^{b}\right)
$$


where $Z^{*}(=R+j X)$ is the impedance, $r_{0}$ and $r_{1}$ are constants with the dimension of resistance, $j$ is $\sqrt{-1}, \omega$ is the angular frequency $(2 \pi f ; f$ :frequency), $\tau$ is a time constant, $b$ is a constant ranging from 0 to 1 . The phase angle $\left(b \times 90^{\circ}\right)$ was almost constant at rest and during secretion in the submandibular gland. The low frequency limit $\left(1 /\left(r_{0}+r_{1}\right)\right)$ was calculated from the data measured at three frequency points, because measured impedances of submandibular gland were expressed as an arc in Cole-Cole plot. Equations (1) and (2) were used to estimate the ECF volume in the submandibular gland (NAKAHARI et al., 1986a, b). That is, the low frequency limit and the conductivity of serum $(11.9 \mathrm{mS} / \mathrm{cm})$ for the maximum estimation, or saline $(15 \mathrm{mS} / \mathrm{cm})$ for the minimum estimation were substituted for $\kappa$ and $\kappa_{\mathrm{m}}$ in Eq. (1), and the ECF volume/total volume ratio $\left(V_{\mathrm{ECF}}\right)$ was calculated as follows:

$$
V_{\mathrm{ECF}}=\left(\kappa / \kappa_{\mathrm{m}}\right)^{2 / 3} \text {. }
$$

In this estimation there are several assumptions, that is, Eq. (1) is applicable to the submandibular gland, the conductivity of the ECF is the same as that of serum or saline, and the volume of the luminal space is negligible because it is less than $3 \%$ of the whole gland (Nakahari, unpublished observations). The increasing or decreasing rate of the ECF volume $\left(\Delta V_{\mathrm{ECF}}(\mu \mathrm{l} /(\mathrm{min} \cdot \mathrm{g}))\right)$ was estimated at various time points and expressed as $\Delta V_{\mathrm{ECF}} / \Delta t$.

In experiments with isolated glands, the ECF volume per initial gland weight, $V_{\text {real-ECF }}(\mathrm{ml} / \mathrm{g})$, was calculated from the value of every $10 \mathrm{~s}$ by the following equation:

$$
V_{\text {real-ECF }}=\left(W_{\mathrm{g}}-W_{\mathrm{s}}\right) \times V_{\mathrm{ECF}} / W_{\mathrm{g}},
$$

where $W_{\mathrm{g}}$ is the initial gland weight, $W_{\mathrm{s}}$ is the cumulative weight of saliva, and $V_{\mathrm{ECF}}$ is obtained from Eq. (3). The intracellular fluid (ICF) volume, $V_{\text {real-ICF }}(\mathrm{ml} / \mathrm{g}$ ), was also calculated from the cellular volume fraction by the following equation:

$$
V_{\text {real-ICF }}=\left(W_{\mathrm{g}}-W_{\mathrm{s}}\right) \times\left(1-V_{\mathrm{ECF}}\right) / W_{\mathrm{g}} .
$$

The decreased ECF volume per initial gland weight, $\delta V_{\mathrm{ECF}}$, is calculated as follows:

$$
\delta V_{\mathrm{ECF}}=V_{\text {initial-ECF }}-V_{\text {real-ECF }} .
$$

The $V_{\text {initial-ECF }}$ is obtained at the start of the experiment from Eq. (3), and $V_{\text {real-ECF }}$ is the value at every $10 \mathrm{~s}$. The fluid stored in the cells, $\left(W_{\mathrm{s}}-\delta V_{\mathrm{ECF}}\right)$, was calculated to compare with the ICF volume.

c) Stereological measurements of ECF volume in submandibular gland. To measure the ECF volume of the submandibular gland at rest and during stimulation after circulatory arrest stereologically, we reported the microscopic structure (NAKAHARI et al., 1985b, 1986b). In the present experiments, the sections of glands were made under four different conditions: at rest, during stimulation, during stimulation after circulatory arrest, and at $5 \mathrm{~min}$ after the cessation of stimulation 
with an intact circulation. Micrographs $(40 \times)$ were traced and subdivided into lobular and extralobular parts, in which the vascular wall and ductal wall were excluded, for stereological analysis. The extralobular parts were measured by an image scanner (AVIO TVIP-2100), and the percentages of extralobular parts were recorded.
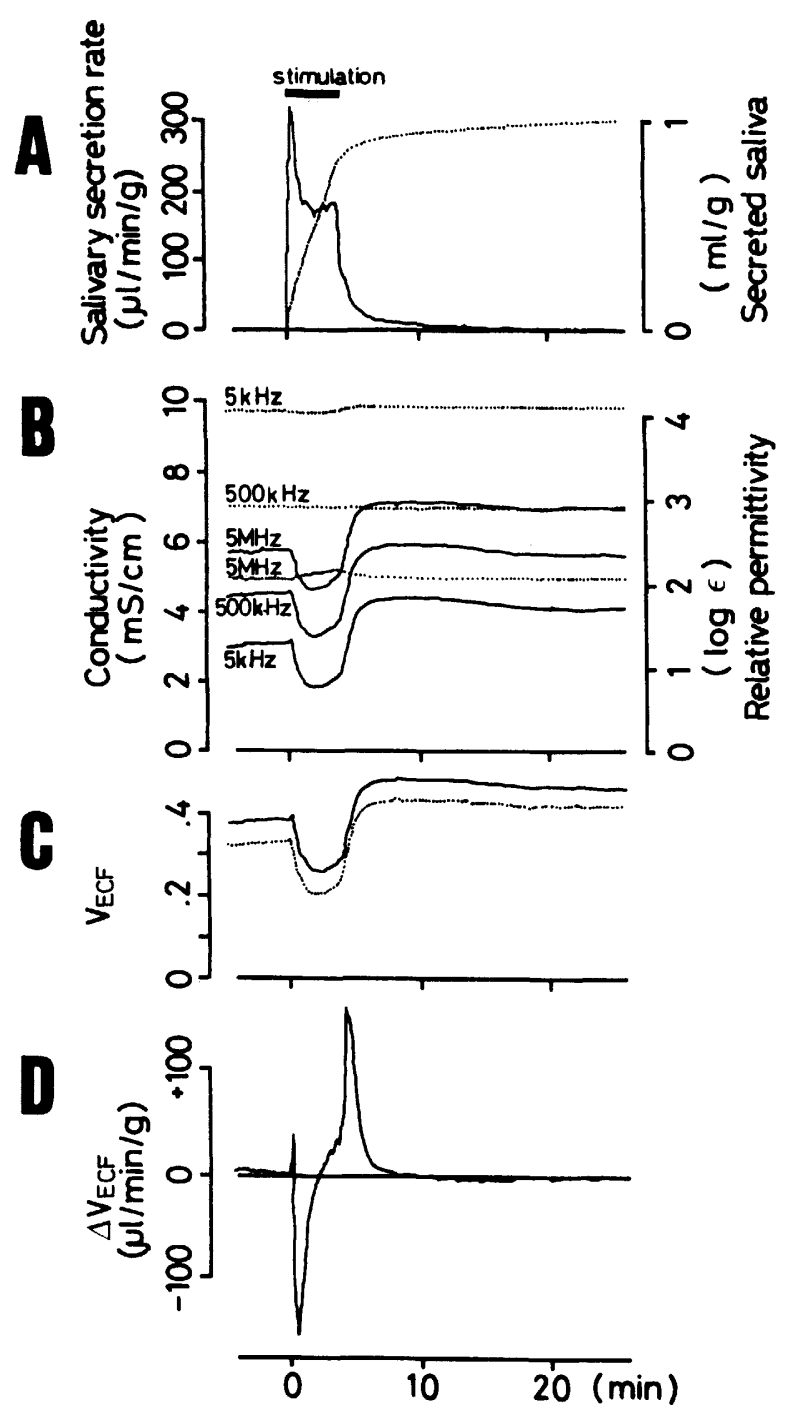

Fig. 1. Experiment in an intact circulation. A: salivary secretion rate (solid line) and secreted saliva (dotted line). B: conductivity (solid line) and relative permittivity (dotted line). C: ECF volume/total volume ratio $\left(V_{\mathrm{ECF}}\right)$. The solid line and dotted line represent the maximum and minimum estimation. D: rate of ECF volume change $\left(\Delta V_{\mathrm{ECF}}\right)$. 


\section{a) Experiments in intact circulation (Figs. 1,2)}

The submandibular gland secretes a large amount of saliva in response to chorda tympani stimulation. In this case, the cumulative amount of saliva secreted during a 4-min stimulation was $0.85 \mathrm{ml} / \mathrm{g}$ with an intact circulation. The continuous measurement of flow revealed that the rate of salivary secretion consisted of two phases. The first was the initial secretion observed within 1 min after the start of stimulation with a high secretory rate and short duration. The second was a steady secretion, following the initial secretion with a constant secretory rate and a long duration (Fig. 1A).

A typical pattern of changes in conductivity and relative permittivity is shown in Fig. 1B. The submandibular gland rapidly lost $40 \%$ of its conductivity at $5 \mathrm{kHz}$, $25 \%$ at $500 \mathrm{kHz}$, and $20 \%$ at $5 \mathrm{MHz}$ with the start of stimulation. This rapid decrease in conductivity coincided with the initial secretion. After the cessation of stimulation, it increased to a higher level than in the prestimulation state, and then it gradually returned to the prestimulation level within $30 \mathrm{~min}$. The relative permittivity change was small at every frequency.

We tried to calculate the ECF volume/total volume ratio from the impedance changes by using Hanai's equation. The changing process of $V_{\mathrm{ECF}}$ is shown in Fig. 1C. The solid line or dotted line shows the maximum or minimum estimation. The negative peak of the changing rate of ECF volume $\Delta V_{\mathrm{ECF}}$ coincides with that of the initial secretion (Fig. 1D, which shows the maximum estimation), that is, the decreasing process of ECF occurred concomitantly with the initial secretion. After the cessation of stimulation, the positive peak of $\Delta V_{\mathrm{ECF}}$ occurred.

Cumulative blood flow also measured continuously (Fig. 2B). This figure was recorded in another experiment. In this case, the rate of blood flow in the gland was

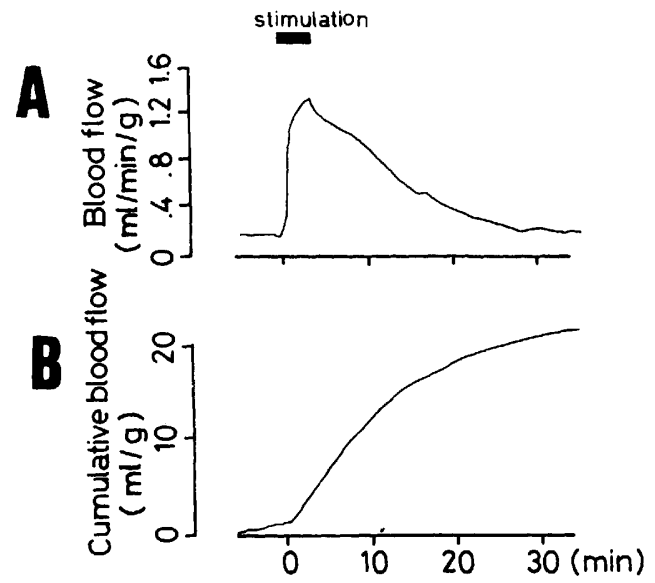

Fig. 2. Blood flow (A) and cumulative blood flow (B) in an intact circulation. 
$2 \mathrm{ml} /(\mathrm{min} \cdot \mathrm{g})$ at rest, and it increased rapidly to $16 \mathrm{ml} /(\mathrm{min} \cdot \mathrm{g})$ in response to the stimulation and decreased gradually after cessation of stimulation (Fig. 2A). However, it remained at a higher level than at the prestimulation level. This high rate of blood flow after stimulation corresponded to the increase of ECF volume.
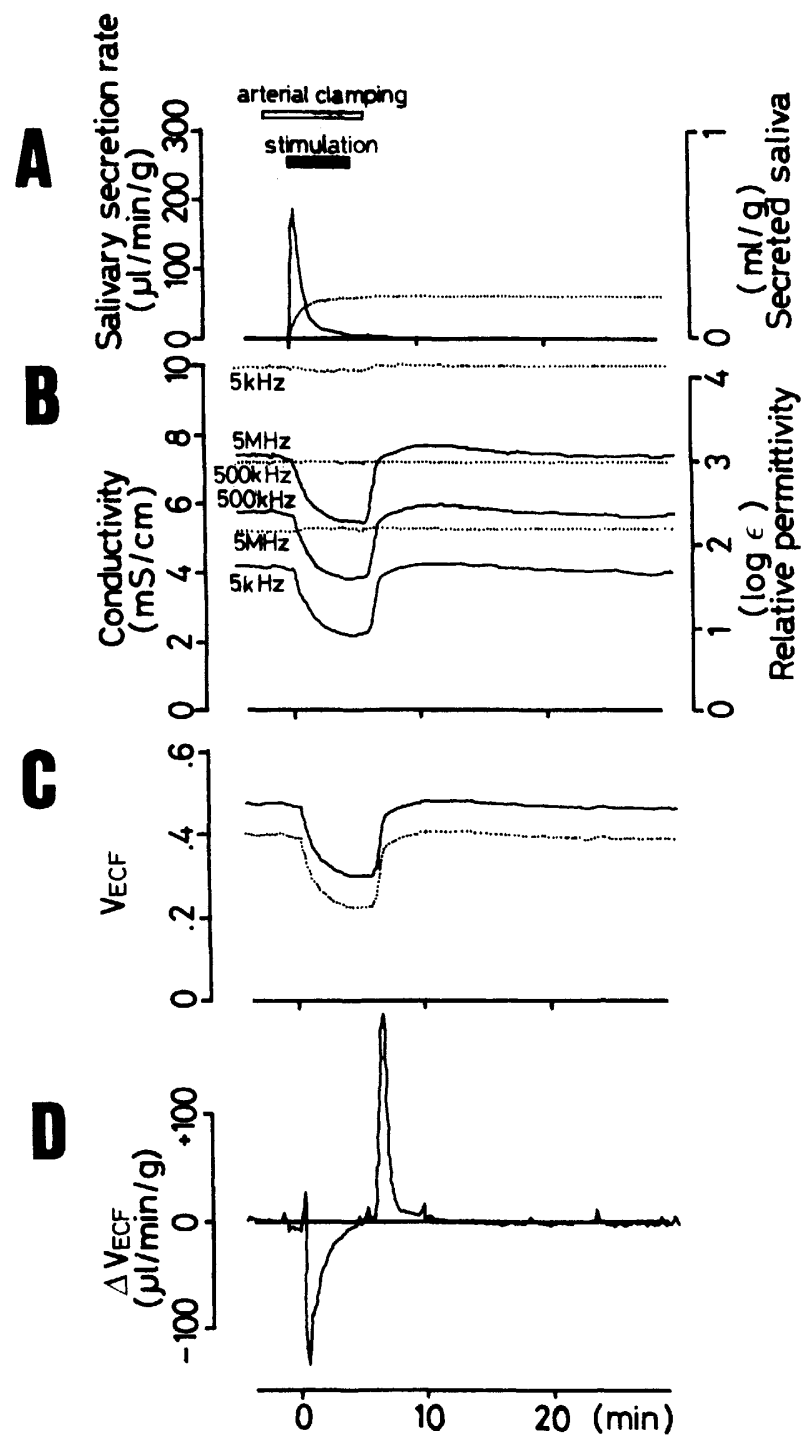

Fig. 3. Experiment after circulatory arrest. A: salivary secretion rate (solid line) and secreted saliva (dotted line). B: conductivity (solid line) and relative permittivity (dotted line). C: ECF volume/total volume ratio $\left(V_{\mathrm{ECF}}\right)$. The solid line and dotted line represent the maximum and minimum estimation. D: rate of ECF volume change $\left(\Delta V_{\mathrm{ECF}}\right)$. 


\section{b) Experiments under condition of arterial clamping (Fig. 3)}

The submandibular gland secreted $0.12 \mathrm{ml} / \mathrm{g}$ of saliva during stimulation, even when the blood flow was arrested by arterial clamping. Under such adverse conditions, the salivary secretory pattern was monophasic, that is, salivary secretion was transient and of short duration; the steady secretion which had a constant secretion rate and a long duration had disappeared (Fig. 3A).

The conductivity of the gland decreased slightly after arterial clamping. The gland rapidly lost $50 \%$ of its conductivity at $5 \mathrm{kHz}, 30 \%$ at $500 \mathrm{kHz}$, and $20 \%$ at $5 \mathrm{MHz}$, with the start of stimulation under this condition (Fig. 3B). The rapid decrease in conductivity also coincided with the initial secretion. After the cessation of the stimulation, the conductivity remained at a low level during arterial clamping. Then, the conductivity recovered upon the removal of arterial clamping and reached a slightly higher level, returning to the prestimulation level in $20 \mathrm{~min}$. The relative permittivity change was small.

The ECF volume/total volume ratio calculated by Eq. (3) is shown in Fig. 3C, in which the solid line or dotted line shows the maximum or minimum estimation. The decrease in $V_{\mathrm{ECF}}$ was more marked than that in an intact blood flow. The negative peak of the changing rate of $\operatorname{ECF}\left(\Delta V_{\mathrm{ECF}}\right)$ coincided with the peak of initial secretion (Fig. 3D, which shows the maximum estimation).

\section{c) Experiment on isolated gland (Fig. 4)}

The isolated gland secreted $0.11 \mathrm{ml} / \mathrm{g}$ of saliva by chorda tympani stimulation, and its secretory pattern was monophasic. That is, a secretion having a transient rate and a short duration occurred (Fig. 4A). The changes of conductivity and relative permittivity were similar to those of glands after circulatory arrest (Fig. 4B).

The weight of the gland was initially $18.65 \mathrm{~g}$ and after the experiments it was $16.60 \mathrm{~g}$. The secreted saliva was $2.05 \mathrm{~g}$. In this case, ECF and ICF volumes were estimated by the value of ECF volume/total volume ratio obtained from impedances. $V_{\mathrm{ECF}}(\%)$ was initially $42 \%$, and it was finally $29 \%$ after stimulation (Fig. $4 \mathrm{C}$, which shows the maximum estimation). Initially, the ECF volume was estimated to be $7.8 \mathrm{ml}(=18.65 \times 0.42)$, and finally $4.8 \mathrm{ml}(=16.60 \times 0.29)$. The volume lost from the ECF was $3.0 \mathrm{ml}(=7.8-4.8)$. This estimation is shown in Fig. $4 \mathrm{C}$ as the real ECF volume per initial gland weight, $V_{\text {real-ECF}}$. The peak in the changing rate of the real ECF $\left(\Delta V_{\text {real-ECF }}\right)$ coincided with that in the initial secretion.

Moreover, the estimation of ICF volume, in which the conductivity of serum $(11.9 \mathrm{mS} / \mathrm{cm})$ was substituted for $\kappa_{\mathrm{m}}$ of Eq. (3), was initially $10.8 \mathrm{ml}(=18.65 \times 0.58)$ and finally it was $11.8 \mathrm{ml}(=16.60 \times 0.71)$. This estimation is shown in Fig. $4 \mathrm{D}$ as the ICF volume, $V_{\text {real-ICF }}$. The volume trapped in the gland, $\left(W_{\mathrm{s}}-\delta V_{\text {real-ECF }}\right)$, is shown in Fig. $4 \mathrm{E}$ and its time derivation, $\Delta\left(W_{\mathrm{s}}-\delta V_{\text {real-ECF }}\right)$, has a peak in the initial secretion. In this estimation, acinar cells of the submandibular gland swelled during stimulation without circulation. 

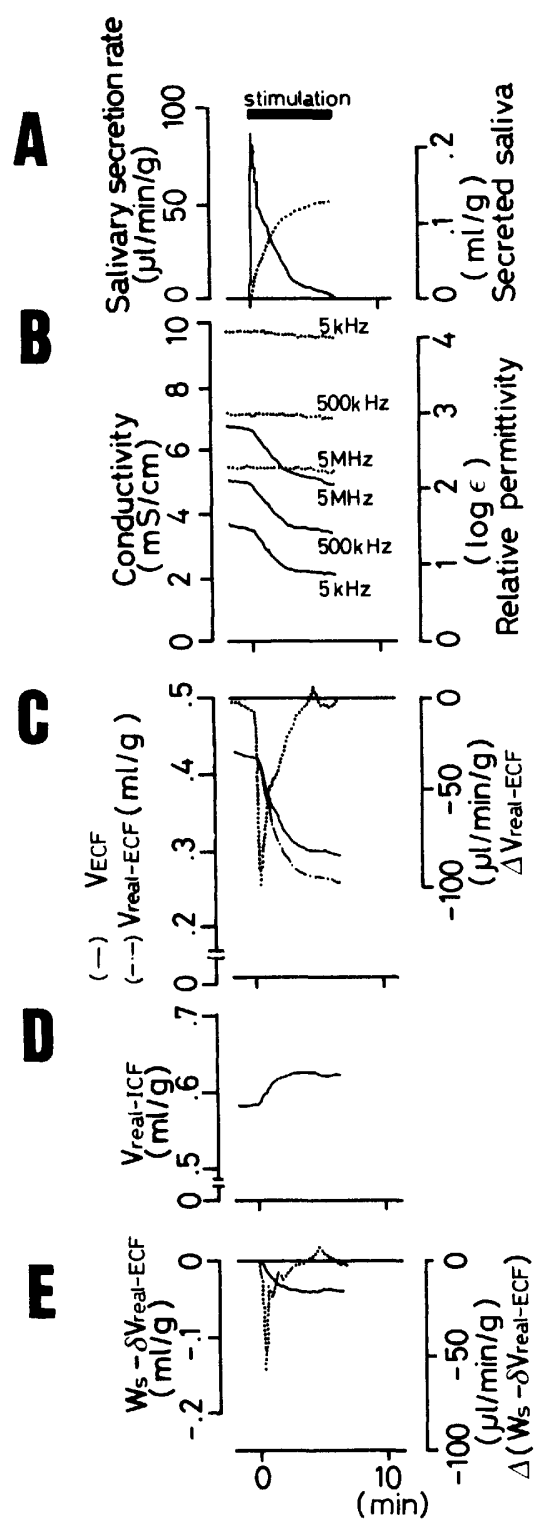

Fig. 4. Experiment on an isolated gland. A: salivary secretion rate (solid line) and secreted saliva (dotted line). B: conductivity (solid line) and relative permittivity (dotted line). C: ECF volume/total volume ratio $\left(V_{\mathrm{ECF}}\right)(-)$, ECF volume per initial gland weight $\left(V_{\text {real-ECF }}\right)(---)$, and rate of real ECF volume change $\left(\Delta V_{\text {real-ECF }}\right)(\cdots)$. D: intracellular fluid volume $\left(V_{\text {real-ICF }}\right)$. E: fluid stored in the glandular cells, $\left(W_{\mathrm{s}}-\delta V_{\text {real-ECF }}\right)$ (solid line) and its changing rate, $\Delta$ $\left(W_{\mathrm{s}}-\delta V_{\text {real-ECF }}\right.$ ) (dotted line). In C, D, E the conductivity of serum $(11.9 \mathrm{mS} / \mathrm{cm})$ was substituted for $\kappa_{\mathrm{m}}$ in Eq. (3) (maximum estimation). 
Table 1. Interlobular space in submandibular glands of dogs measured by stereological method.

\begin{tabular}{|c|c|}
\hline Condition & Mean \pm S.E. $(\%)$ \\
\hline $\begin{array}{l}\text { Resting state } \\
\quad \text { with an intact circulation }\end{array}$ & $17.86 \pm 1.76 \quad(n=36)$ \\
\hline $\begin{array}{l}\text { During stimulation } \\
\text { with an intact circulation }\end{array}$ & $10.61 \pm 0.71 * \quad(n=36)$ \\
\hline $\begin{array}{l}\text { During stimulation } \\
\text { after circulatory arrest }\end{array}$ & $6.53 \pm 0.46^{* *}(n=35)$ \\
\hline $\begin{array}{l}\text { After cessation of stimulation } \\
\text { with an intact circulation }\end{array}$ & $24.13 \pm 1.99^{* *}(n=34)$ \\
\hline
\end{tabular}

The intralobular space changes little and its value is $14-15 \%$ in every condition. Total ECF volume is the sum of two values, inter- and intralobular space. *Value is significantly different $(p<0.005)$ from corresponding value of resting state. ${ }^{* *}$ Value is significantly different $(p<0.001)$ from corresponding value of resting state.

\section{d) Histological observation (Fig. 5)}

The ECF of the submandibular gland was classified in two compartments: intralobular and interlobular spaces, as shown in a previous report (NAKAHARI et al., 1985b). The interlobular space was wide in the resting state (Fig. 5A). During stimulation of an intact circulation, it was narrow (Fig. 5B). It became dense threadlike lines by the stimulation during arterial clamping (Fig. 5C). After the cessation of stimulation with an intact circulation, it was widened more than in the prestimulation state (Fig. 5D). The decrease in the ECF space during stimulation after arterial clamping has been reported in the interlobular space (NAKAHARI et al., 1985b). The present study showed the interlobular space with an intact circulation to be narrow during stimulation and to be wide after cessation of stimulation.

A stereological study was also performed. The measured interlobular spaces, excluding vascular and ductal walls, are shown in Table 1. The intralobular ECF space was $14-15 \%$ at rest and during stimulation under arterial clamping condition (NAKAhari et al., 1986b) and it changed little.

\section{DISCUSSION}

The present study revealed the dynamic changes in ECF by measuring the impedance of submandibular gland at the intervals of $10 \mathrm{~s}$. The continuous measurements could be done by the selection of only three frequencies $(5 \mathrm{kHz}$, $500 \mathrm{kHz}, 5 \mathrm{MHz})$ within a wide frequency range $(5 \mathrm{~Hz}-13 \mathrm{MHz})$, and it revealed not only the decrease but also increase in ECF volume in secretory processes. It was found that ECF volume changes were closely related to the conditions of submandibular gland, such as secretory stimulation, blood flow, blood pressure of artery and vein. 

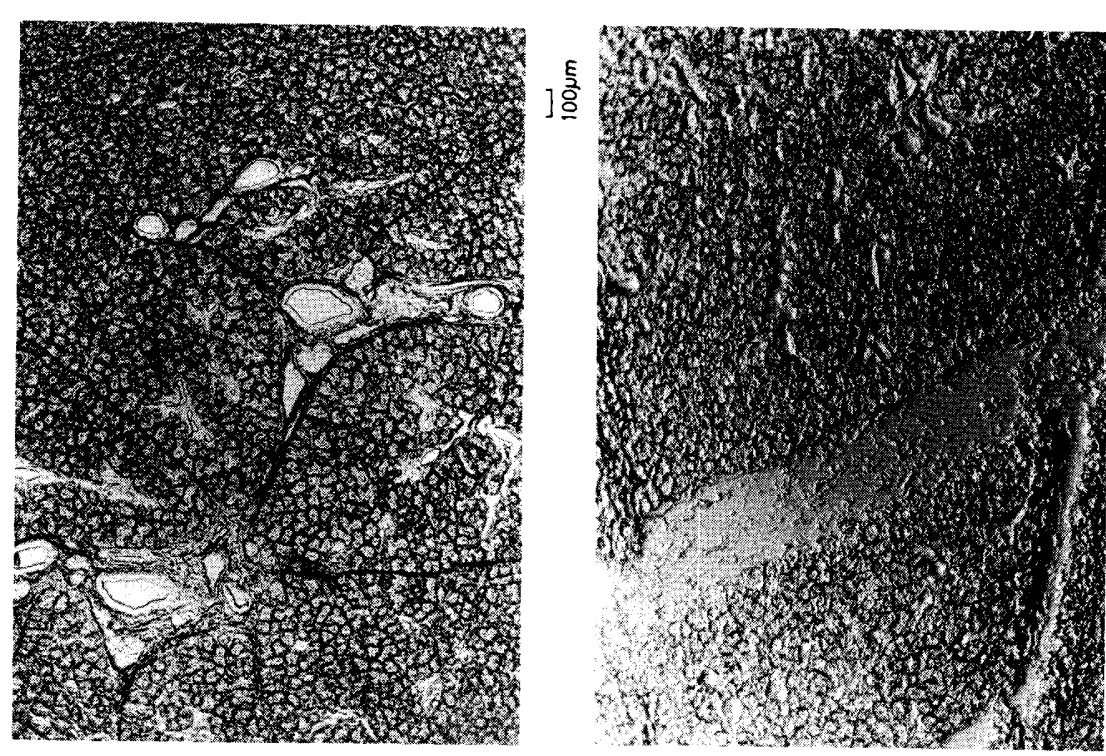

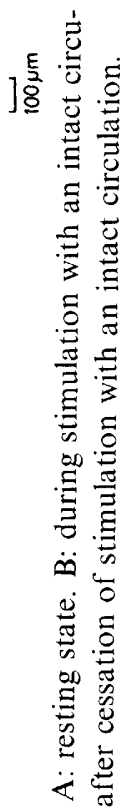

$3+70$ \%

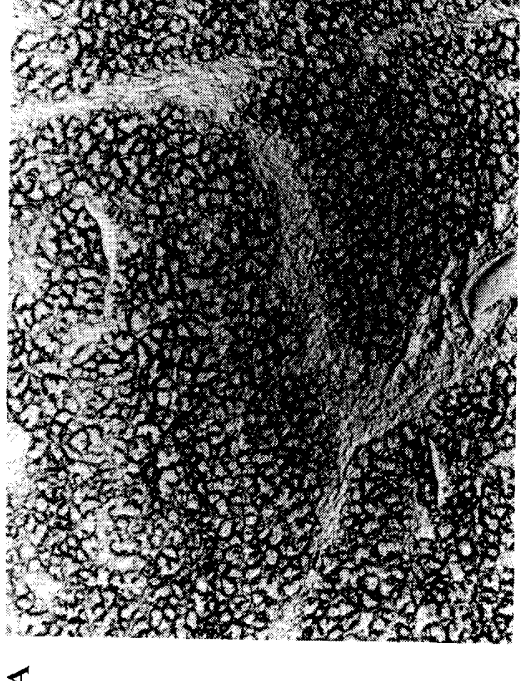

0

$\ddot{g} \ddot{0}$

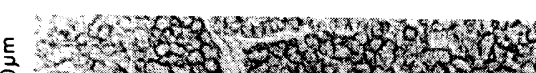

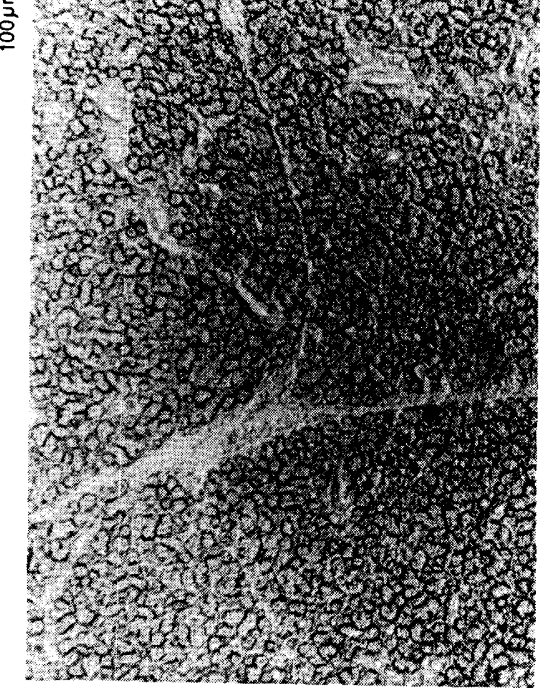

]

$\frac{\pi}{3}$

을

节

氝

을

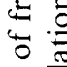

竞

政

:

छ

롱

ป

in

is 
There are some difficulties in impedance measurements of the submandibular gland. The electrode polarization in low frequencies, especially less than $1 \mathrm{kHz}$, affected the measured impedances. In the present study, the impedances of submandibular glands were measured at three frequencies $(5 \mathrm{kHz}, 500 \mathrm{kHz}$, and $5 \mathrm{MHz}$ ) and no correction for the effects of electrode polarization was made. However, to check the effects of electrode polarization, the impedances of submandibular gland were measured in 131 frequency points scanned in the log-linear range of $5 \mathrm{~Hz}-13 \mathrm{MHz}$. The effect of electrode polarization has been corrected by the substitution technique (SCHWAN, 1963), and the data thus calculated were fitted to Eq. (2) using the least-squares method. Using the same data without the correction for the effect of electrode polarization, the low frequency limit of the conductivities was also calculated from the data of $5 \mathrm{kHz}, 500 \mathrm{kHz}$, and $5 \mathrm{MHz}$. The low frequency limit of conductivity calculated at three frequencies was compared with that of wide range, and it was estimated to be smaller due to the electrode polarization. But its extent was less than $10 \%$, thus $V_{\mathrm{ECF}}$ was underestimated to be less than 0.03 lower than that estimated within a wide frequency range. Furthermore, the electrode polarization changed in every measurement although its change was small, because the electrodes were attached to the submandibular gland directly, and the correction of electrode polarization effect from the data of only three frequencies was difficult.

In the present experiments, the conductivity of ECF was not measured directly. The ionic concentration of the interstitial fluid was assumed to be the same as that of the serum, but its protein concentration $(2 \mathrm{~g} / \mathrm{dl})$ was lower than the serum. The saliva in the acinus seems to be isotonic (Young and VAN LENNEP, 1978), but that of ductal space seems to be hypotonic. Thus, the conductivities of these fluid compartments were not the same. But as the luminar space was less than $3 \%$, its effects would be negligible. Therefore the conductivity of ECF would be higher than serum, and lower than saline. If the conductivity of the ECF was assumed to be same as that of the serum $(11.9 \mathrm{mS} / \mathrm{cm})$, the ECF volume/total volume ratio, $V_{\mathrm{ECF}}$, was the maximum estimation. IF the conductivity of the ECF was the same as saline $(150 \mathrm{mM} \mathrm{NaCl}: 15 \mathrm{mS} / \mathrm{cm})$, the value of $V_{\mathrm{ECF}}(\%)$ was $85 \%$ that of the serum, and was the minimum estimation. The ECF volume/total volume ratio calculated from Eq. (3) is shown in Table 2 as maximum and minimum estimations. Their values corresponded with those in the stereological study.

The impedance method is sensitive to the changes in ECF volume, and is useful for continuous monitoring of ECF or cellular volume. However, there is no suitable model for organs having complicated structures such as the salivary gland, pancreas, etc. In this report we assumed the theory developed for concentrated cellsuspension systems to be applicable in estimating the ECF volume of the submandibular gland. We found that our estimations on ECF obtained from impedances reflect roughly but sensitively the changing processes in ECF volume because the stereological study of submandibular gland supports our estimation. We think that this estimation is useful as an index of extracellular volume/total volume ratio. 
Table 2. Maximum and minimum estimation of (ECF volume)/(total volume) ratio, $V_{\text {ECF }}(\%)$, in submandibular glands of dogs calculated by Hanai's equation.

\begin{tabular}{lcc}
\hline Condition & \multicolumn{2}{c}{ Mean \pm S.E. $(\%)$} \\
\cline { 2 - 3 } & $38.94 \pm 1.15$ & $33.10 \pm 1.06 \quad(n=9)$ \\
\hline $\begin{array}{l}\text { Resting state } \\
\text { with an intact circulation } \\
\text { During stimulation } \\
\quad \text { with an intact circulation } \\
\text { During stimulation } \\
\quad \text { after circulatory arrest } \\
\text { After cessation of stimulation } \\
\text { with an intact circulation }\end{array}$ & $29.38 \pm 0.75^{*}$ & $25.00 \pm 0.69^{*}(n=8)$ \\
& $24.43 \pm 0.83^{*}$ & $20.77 \pm 0.76^{*}(n=7)$ \\
\end{tabular}

*Value is significantly different $(p<0.001)$ from corresponding value of resting state.

A decrease in conductivity in secreting salivary glands has been reported (VAN HarreVeld et al., 1961). VAN Harreveld et al. (1961) reported that the decrease in tissue conductivity was caused by the decrease of extratubular ions. Our study revealed the decrease of ECF space. Therefore the decrease in conductivity was caused by a reduction of the ECF volume and not by a reduction of the extratubular ionic concentration.

The conductivity increased more after the cessation of stimulation in an intact circulation than in the prestimulation, as shown in Fig. 1B. This increase refers to the increase in the ECF space and was also detected histologically as the increase in the interlobular space. On the other hand, the blood flow also maintained a 3-5 times higher level after cessation of stimulation than in the prestimulation, and then it is assumed that the high blood flow induced edema in the interlobular space.

The impedance of isolated glands provided the information of cellular volume. In this condition, though the gland was anoxic and had no fluid supply, it was estimated that the cell swelled about $5 \%$ during secretion. The process of cellular volume swelling corresponded with the salivary secretion and ECF volume decrease. These findings propose that fluid secreted in the acinus is transported via a cellular pathway as well as a paracellular pathway in the initial secretion.

Continuous measurement of salivary secretion revealed that the secretion occurred in two phases: an initial secretion and a steady secretion (MURAKAMI et al., 1980). An initial secretion occurs within the first minute after the start of stimulation and has transient and high secretory rates. This was observed even in circulatory arrest. A steady secretion continues during stimulation at a constant rate, and diminishes after circulatory arrest. These two processes in salivary secretion have been discussed concerning the energy processes. That is, the heat production was less in the initial secretion than in the steady secretion and the $\mathrm{O}_{2}$ uptake was also less in the normal circulation. The heat production was also 
transient, like the secretion in circulatory arrest. The initial secretion was suggested to be driven by stored electrochemical energy and the steady secretion to be driven by active transport coupled with a chemical reaction such as Na-K ATPase (MuraKami, 1981; Michigami, 1985). In our present observation, the decrease in conductivity also occurred in the initial secretion and the decrease in conductivity indicated a reduction in the ECF volume as shown in the histological study. Therefore, in the initial secretion, the fluid secreted as saliva is supplied from the fluid stored in the ECF space, and in the steady secretion it is supplied from the blood.

The ECF volume of the salivary gland has been measured using the space method by many investigators (SCHNEYER and SCHNEYER, 1960; IMAI, 1965; Macknight and Leaf, 1977; Pirani et al., 1987). Their measurements were performed in vitro and were useful in an ionic distribution study. But, the ECF volume in vivo is necessary to consider dynamic process of secretion, because the amount of ECF changes under various conditions, such as stimulation, blood flow and anoxia, as shown in this study. When the gland was removed from the animal and blotted to remove edema fluid to measure the inulin space, these procedures were the possible causes of the small ECF volume. Although our estimations in the present study are greater than those in their reports, they reflect the increase or decrease in the ECF volume of the submandibular gland under vaious conditions. Though further studies are required on the estimation of ECF volume, we think that the quantity of ECF in the submandibular gland in vivo is greater than that estimated by the inulin space method in vitro.

We thank Mr. T. Takeuchi (student at our school) for his technical support in microcomputer programs and Dr. M. Murakami (National Institute for Physiological Science) for his useful suggestions and discussion. This work was supported in part by a Grant-in-Aid for Scientific Research (No. 59570045) from the Ministry of Education, Science and Culture, Japan.

\section{REFERENCES}

Asami, K. and IRIMAJirI, A. (1984) Dielectric dispersion of a single spherical bilayer membrane in suspension. Biochim. Biophys. Acta, 769: 370-376.

Asami, K., Irimajiri, A., Hanai, T., Shiraishi, N., and Utumi, K. (1984) Dielectric analysis of mitochondria isolated from rat liver. I: Swollen mitoplasts as simulated by a singleshell model. Biochim. Biophys. Acta, 778: 559-569.

ColE, K. S. (1968) Membranes, Ions and Impulses, University of California Press, Berkeley and Los Angeles, pp. 6-59.

COLE, K.S. and COLE, R. H. (1941) Dispersion and absorption in dielectrics I: Alternating current characteristics. J. Chem. Phys., 9: 341-351.

Hanal, T. (1968) Electrical Properties of Emulsions. In: Emulsion Science, ed. by Sherman, P., Academic Press, London and New York, pp. 353-478.

IMAI, Y. (1965) Study of the secretion mechanism of the submaxillary gland of dog. Part 2. Effect of exchanging ions in the perfusate on salivary secretion and secretory potential 
with special reference to the ionic distribution in the gland tissue. J. Physiol. Soc. Jpn., 27: 313-324 (in Japanese).

Irimajiri, A., HanaI, T., and INOUYE, A. (1975) Evaluation of a conductometric method to determine the volume fraction of the suspension of biomembrane-bounded particles. Experientia, 31: 1373-1374.

Macknight, A. D. C. and Leaf, A. (1977) Regulation of cellular volume. Physiol. Rev., 57: $510-573$.

Michigami, M. (1985) Effect of oxygen supply and potassium on salivary secretion by submandibular glands of dogs during constant flow perfusion. J. Osaka Med. Coll., 44: 271-279 (in Japanese).

Murakami, M., Mori, H., NaKahari, T., and Imai, Y. (1980) Method and application of weight differentiating flowmeter. Jpn. J. Physiol., 30: 791-794.

Murakami, M. (1981) Heat production, blood flow, $\mathrm{O}_{2}$ uptake and $\mathrm{CO}_{2}$ output in the secretory process of the dog submandibular gland. J. Physiol. Soc. Jpn., 43: 135-147 (in Japanese).

Nakahari, T., Miyamoto, M., Sano, K., Yoshida, H., and Imai, Y. (1985a) Decrease of extracellular fluid in dog submandibular glands during secretion under arterial clamping condition. Jpn. J. Physiol., 35: 1085-1090.

Nakahari, T., Seo, Y. Murakami, M., Mori, H., Miyamoto, S., Imai, Y., and Watari, H. (1985b) ${ }^{31}$ P-NMR study of dog submandibular gland in vivo and in vitro using the Topical Magnetic Resonance. Jpn. J. Physiol., 35: 729-740.

NAKAHARI, T., Yoshida, H., and IMAI, Y. (1986a) Impedance analysis of ECF in salivary gland. Biomed. Res., 7 (Suppl. 2): 221-224.

Nakahari, T., Yoshida, H., MiYamoto, M., and Imai, Y. (1986b) Measurement of extracellular fluid change in salivary gland using an impedance method. Jpn. J. Physiol., 36: 565-583.

Pirani, D., Evans, L. A. R., Cook, D. I., and Young, J. A. (1987) Intracellular pH in the rat mandibular salivary gland: The role of $\mathrm{Na}-\mathrm{H}$ and $\mathrm{Cl}-\mathrm{HCO}_{3}$ antiports in secretion. Pflügers Arch., 408: 178-184.

SCHNEYER, L. H. and SCHNEYER, C. A. (1960) Electrolyte and inulin spaces of rat salivary gland and pancreas. Am. J. Physiol., 199: 649-652.

Schwan, H. P. (1963) Electrophysiological methods, Part B. In: Physical Techniques in Biological Research, ed. by Nastuk, W. C., Academic Press, New York, Vol. 6B, pp. 232-407.

Van Harreveld, A., Potter, R. L., and Sloss, L. J. (1961) Electrical conductivity and electrolyte distribution in secreting salivary gland. Am. J. Physiol., 201: 1002-1006.

Young, J. A. and VAN LenNeP, E. W. (1978) Transport in salivary and salt glands. In: Membrane Transport in Biology IV B Transport Organs, ed. by GIEBISH, G., TosTesON, D. C., and Ussing, H. H., Springer-Verlag, Berlin Heidelberg, Vol. 4B, pp. 563-692. 